\title{
Cochrane review summary: education for contraceptive use by women after childbirth
}

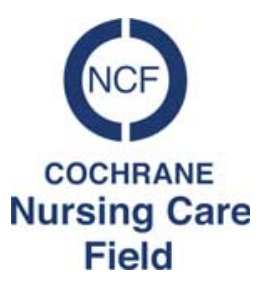

\section{Daksha Trivedi}

Senior Research Fellow, Evidence Based Practice, Centre for Research in Primary and Community Care, University of Hertfordshire, Hatfield, UK

Key words: contraception; counselling; education; family planning; intervention; postpartum

\section{Review question}

What are the effects of educational programmes about contraceptive use for postpartum mothers?

\section{Relevance to primary care nursing}

Nurses who work in primary care provide a range of contraceptive services, including contraceptive education in family planning consultations. The National Institute for Clinical Excellence (NICE) guidelines recommend that contraceptive methods and advice for women after childbirth is discussed early in the postpartum period and is an integral part of postpartum check in primary care (NICE, 2006), which is usually delivered by community midwives. At present, there is a lack of evidence on the effectiveness of postpartum contraceptive education. This systematic review has summarised all the available evidence of programmes delivered by various health professionals including physicians, nurses and midwives (Lopez et al., 2012).

\section{Type of review}

This is a summary of a Cochrane review containing 10 randomised controlled trials (RCTs) from five countries, assessing the effects of educational interventions on unplanned pregnancies and choice or use of contraception. Meta-analysis was not

Correspondence to: Dr Daksha Trivedi, PhD., MPhil (Cantab), MSc, BSc (Hons), Senior Research Fellow, Evidence Based Practice, Centre for Research in Primary and Community Care, University of Hertfordshire, College Lane, Hatfield AL10 9AB, UK. Email: d.trivedi@herts.ac.uk

(C) Cambridge University Press 2013 performed because of varied study designs (Lopez et al., 2012).

\section{Characteristics of the evidence}

The review included a total of 3505 participants. The participants were all women giving birth at 20 weeks' gestation or more. Studies that addressed the needs of women with alcohol or drug problems or women with chronic health conditions, such as HIV or diabetes, were excluded. Interventions needed to have examined postpartum education about the use of contraception, including lactational amenorrhea, and be provided within one month of delivery. Educational interventions included written materials, video or audio recordings, or individual or group counselling. Interventions on postpartum contraception that commenced during the prenatal period were not examined.

The primary outcomes were unplanned pregnancy and contraceptive use. Additional outcomes included knowledge about contraception, breastfeeding and satisfaction with postnatal care.

The interventions varied in the content, format and duration of the education and included oral contraceptive use, parenting, educational support, including breastfeeding and infant care, reproductive health, family planning, structured support and counselling, including motivational interviewing. They were provided during the postpartum hospital stay, or two or three weeks later. They were compared with either no intervention, or usual or alternative care. In most trials, trained clinicians provided the education, although the intensity of training varied. 
The trials were categorised into two groups according to the type of intervention: four provided counselling with one or two contacts, and six provided programmes with multiple contacts delivered at home, clinic or by phone. Three studies of 1010 women based in a hospital setting assessed counselling in a single session on contraception. Of these, one provided informal counselling regarding contraception, in the presence of the husband or a close relative, to 648 women. A didactic approach was used with an outline for talking points, and a pamphlet provided on contraceptive methods. The control group had no counselling or pamphlet provided. Another compared a video presentation with educational literature or a physician-patient counselling session in 329 women. The third study compared a multi-component intervention consisting of counselling, a videotape about oral contraceptives and written material versus resident-physician counselling (usual care) provided to 33 women post delivery by physicians and nurses. The fourth study provided up to two health education sessions and compared four groups: two received health education before discharge from hospital. One of these had a second session at home three months post delivery. A third group received health education at three months and the fourth group had no education. The programme was delivered to 540 women by trained health educators, midwives and a community health worker. It included one-to-one health education sessions that were interactive and supportive covering infant care, contraception, family planning and exclusive breastfeeding. The follow-up times varied from eight weeks to 12 months.

The remaining six trials provided programmes with multiple contacts delivering broader health issues including contraception, of which five targeted adolescents $(n=1049)$. One study included 181 adolescents and focused on delaying second births, parenting and contraception (mean 6.63 home visits) compared with usual care. The intervention was delivered by college students from participants' backgrounds. Another provided six structured home visits to 139 teenagers, in addition to routine postnatal support delivered by nurse midwives. One study of 903 women compared four home visits by registered midwives (intervention included breastfeeding and family planning) with one visit (no family planning) or no visit. Computerassisted motivational intervention (CAMI) plus multi-component intervention (parent training and case management) was evaluated in 237 pregnant adolescents, compared with CAMI only and usual care with a maximum of nine quarterly sessions delivered at home by paraprofessional women from participants' communities.

One clinic-based study on 243 teenagers evaluated multiple clinic contacts from specialist services within the well-baby clinic (enhanced care) compared with usual well-baby care. The intervention included contraception, mother's return to school, infant health and reminder calls and letters for appointments. It was delivered by a social worker, paediatrician, nurse practitioners and volunteers. Another study of 249 pregnant or newly parenting teens delivered multiple cell phone sessions on reproductive health planning for 18 months of counselling sessions (maximum 42), and quarterly group sessions compared with usual health education services provided through schools and health-care facilities. In a few studies, the women who provided the intervention had demographics similar to those of the participants.

The number of visits, frequency and duration of the interventions varied, and follow-up period ranged from four months to 24 months.

\section{Summary of key evidence}

The included trials delivered various types of postpartum educational interventions in various settings, some during postpartum hospital stay and others a few weeks later. Six studies were graded as moderate or high quality and four as low or very-low quality. Two trials did not contribute to the findings because of insufficient data or an inadequate sample size. Of the five trials that showed intervention effectiveness, only two were of moderate or high quality and three trials were of low or very-low quality. Therefore, the overall evidence of effect was of low to moderate quality. Effect sizes are shown as odds ratio (OR) with $95 \%$ confidence intervals (CI) for interventions showing a significant effect.

\section{Counselling (one or two contacts)}

Of the four trials providing one or two counselling session, one delivering a single informal session showed that women receiving counselling were more likely than those without counselling to use contraception at $8-12$ weeks postpartum 
(OR 19.56; 95\% CI 11.65-32.83), although the assessments were short term. More women in the counselling group planned to use a modern contraceptive method by six months postpartum compared with a control group (OR 1038.09; 95\% CI 64.15-6799.73). One study reported satisfaction from physician-patient counselling (OR 0.27; 95\% CI 0.07-0.98) but did not provide sufficient data on contraceptive use. Another study evaluating a multi-component intervention did not have adequate sample size to detect significant differences over 12 months.

In the fourth study providing up to two sessions, women with an immediate postpartum session before discharge were more likely to use contraception than the group with no immediate session at six months (OR 1.62; 95\% CI 1.06-2.50) but did not differ at three months. Other comparisons did not show evidence of effectiveness in contraceptive use: two sessions compared with one immediate session or the later session compared with no education. There were no significant differences in exclusive breastfeeding, which was emphasised in the immediate postpartum session.

\section{Programmes with multiple contacts (home, phone or clinic)}

Six trials provided multifaceted programmes with multiple contacts in various settings where family planning education was integrated with other health education or health services.

Three studies showed evidence of effectiveness, of which one reported that adolescents who received multiple home visits over two years were less likely to have had a second birth assessed at home visits compared with those receiving usual care (OR 0.41; $95 \%$ CI $0.17-1.00)$. The second study reported that teenagers in a structured home-visiting programme were more likely to use contraception at six months than those who had standard home visits (OR 3.24; 95\% CI 1.35-7.79). This study also reported an improvement in contraceptive knowledge at six months in the intervention group (reported mean difference $0.92 ; 95 \%$ CI $0.32-1.52$ ), although the review did not examine this because of insufficient data. The third study showed that adolescents receiving enhanced well-baby care were less likely to have a repeat pregnancy by 18 months (assessed by self-report) compared with usual well-baby care (routine care) (OR 0.35; 95\% CI 0.17-0.70).
The other three studies showed no evidence of effectiveness. Of these, CAMI, with or without parenting curriculum for pregnant adolescents, showed a non-significant reduction in repeat births by 24 months (assessed through records) compared with the usual care group. One study involving four home visits showed no significant effect on contraceptive use or self-reported pregnancy compared with usual care that did not have a home visit. Another study evaluating cell phone counselling in adolescents reported no significant effect on pregnancy rates during the two-year follow-up (confirmed by urine tests).

\section{Implications for practice}

Although half of the interventions were effective in reducing repeat pregnancies or births and increasing contraceptive use, the overall evidence of effectiveness was of low to moderate quality. Two trials of counselling providing one or two sessions were effective but were limited by self-reported outcomes and short-term outcome assessment. Three trials of multifaceted programmes involving multiple contacts were effective, but their applicability in other locations is uncertain and health-care providers would need to consider which intervention might be appropriate for their setting and level of resources.

\section{Implications for research}

High-quality trials are required to establish intervention effectiveness. Self-reported outcomes need to be validated with other sources and different interventions examined in RCTs with longer followup for outcome assessment. Five trials showing positive effects were conducted in the United States, Australia, Nepal and Pakistan, and the programmes need to be further evaluated in other settings and locations.

\section{Acknowledgements}

The author is a member of the Cochrane Nursing Care Field (CNCF). Financial support: This research received no specific grant from any funding agency, commercial or not-for-profit sectors. Statement of interest: None. Ethical standards: Not applicable. 


\section{Daksha Trivedi}

This is a summary based on secondary research and is not dealing with animals.

\section{References}

National Institute for Clinical Excellence (NICE). 2006: Clinical guideline. 37. Routine postnatal care of women and their babies. Developed by the National Collaborating Centre for Primary Care; Issued July 2006; http://www.nice. org.uk/nicemedia/pdf/CG37NICEguideline.pdf

Lopez, L.M., Hiller, J.E., Grimes, D.A. and Chen, M. 2012: Education for contraceptive use by women after childbirth. Cochrane Database of Systematic Reviews 2012, Issue 8. Art. No.: CD001863. DOI: 10.1002/14651858. CD001863.pub3. 